Published in final edited form as:

Gynecol Oncol. 2016 October ; 143(1): 18-21. doi:10.1016/j.ygyno.2016.07.116.

\title{
Impact of postoperative intensity-modulated radiation therapy (IMRT) on the rate of bowel obstruction in gynecologic malignancy
}

\author{
Karin K Shih ${ }^{\mathrm{a}}$, Carla Hajj ${ }^{\mathrm{b}}$, Marisa Kollmeier ${ }^{\mathrm{b}}$, Melissa K Frey ${ }^{\mathrm{c}}$, Yukio Sonoda ${ }^{\mathrm{d}, \mathrm{e}}$, Nadeem R \\ Abu-Rustum $^{\mathrm{d}, \mathrm{e}}$, and Kaled M Alektiar ${ }^{\mathrm{b}}$ \\ aGynecologic Oncology, Women's Comprehensive Health Center, Northwell Health Physician \\ Partners, Manhasset, NY, USA

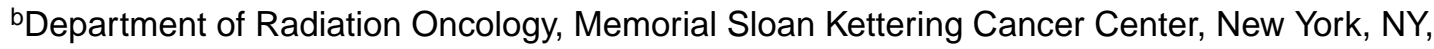 \\ USA \\ 'New York University Langone Medical Center, New York, NY, USA \\ 'Gynecology Service, Department of Surgery, Memorial Sloan Kettering Cancer Center, New \\ York, NY, USA \\ eDepartment of Obstetrics and Gynecology, Weill Cornell Medical College, New York, NY, USA
}

\section{Abstract}

Objective-The purpose was to determine the potential impact of IMRT on the rate of bowel obstruction (BO), in patients with gynecologic malignancies undergoing postoperative pelvic RT.

Methods-We performed a retrospective review of all patients with endometrial or cervical cancer who received postoperative pelvic RT at our institution from 2000-2012. Patients who received definitive or palliative $\mathrm{RT}$, or those with $\mathrm{BO}$ due to disease progression, were excluded. Standard two-sided statistical tests were used to evaluate for associated risk factors. Kaplan-Meier, Log rank and Cox proportional hazards regression analysis tests were performed for actuarial analysis.

Results-A total of 224 patients were identified, 120 (54\%) received postoperative pelvic IMRT and 104 (46\%) 3-dimentional (3-D) RT. Median follow-up time was 67 months. BO was grade 1 (asymptomatic) in $2 / 228(0.9 \%)$, grade 2 (conservative management) in $4(1.8 \%)$, and grade $3 \geq$ in $4(1.8 \%)$. Overall, the 5-year actuarial rate of BO was $4.8 \%$. The 5-year rate of BO in the IMRT group was $0.9 \%$ compared to $9.3 \%$ for 3 -D RT ( $P=0.006$ ). Patients with BMI $\geq 30 \mathrm{~kg} / \mathrm{m}^{2}$ were less

Corresponding author: Kaled M. Alektiar, MD, Department of Radiation Oncology, Memorial Sloan Kettering Cancer Center, 1275 York Avenue, New York, NY 10065, 212-639-7981, alektiak@mskcc.org.

Publisher's Disclaimer: This is a PDF file of an unedited manuscript that has been accepted for publication. As a service to our customers we are providing this early version of the manuscript. The manuscript will undergo copyediting, typesetting, and review of the resulting proof before it is published in its final citable form. Please note that during the production process errors may be discovered which could affect the content, and all legal disclaimers that apply to the journal pertain.

Conflict of Interest Statement

The authors have no conflicts of interest to disclose 
likely to develop BO ( $2.6 \%$ vs. $8.3 ; P=0.03)$. On multivariate analysis, only IMRT retained its significance as an independent predictor of less BO ( $\mathrm{p}=0.022)$.

Conclusions-The use of postoperative IMRT for cervical and endometrial cancer was associated with significant reduction in the rate of bowel obstruction. This difference maintained its statistical significance on multivariate analysis. Such finding if confirmed by others will help further solidify the benefit of IMRT in gynecologic cancers.

\section{Introduction}

Because the gastrointestinal epithelium has a high proliferative rate, it is especially susceptible to the effect of pelvic radiation therapy (RT). The majority of gastrointestinal toxicity related to pelvic RT is acute and can be effectively managed conservatively with bowel rest and/or pharmacologic agents. Bowel obstruction (BO), on the other hand, is a serious gastrointestinal complication that often requires hospitalization and may warrant surgical intervention. If left untreated, significant morbidity can result from BO such as bowel perforation, peritonitis, sepsis, bowel ischemia, or even death. The pathogenesis is related to direct radiation injury to mucosal stem cells within the crypts of Lieberkuhn or the result of microvascular damage. This vasculitis results initially in ischemia and inflammation, followed by fibrosis of the mucosa. [1] In turn this fibrosis results in decreased bowel motility. As such, the use of postoperative pelvic RT is associated with an increased risk of BO. This has been demonstrated in gynecologic malignancies, as well as other pelvic malignancies such as colorectal carcinoma. [2-4]

Compared with conventional 3-dimentional (3-D) RT, intensity modulated radiation therapy (IMRT) allows for delivery of highly conformal therapeutic radiation doses to oncologic targets while constraining the dose to healthy tissue. Several studies have demonstrated the dosimetric advantages of pelvic IMRT over conventional 3-D RT in gynecologic cancers as well as its clinical feasibility. [5-7] More importantly many investigators demonstrated that pelvic IMRT has a significant impact on reducing hematological toxicity when compared to 3-D RT [8,9], as well as better morbidity profiles for the genitourinary and gastrointestinal tracts. [10-12]

However, one area that has received little attention is the potential impact of postoperative pelvic IMRT on the risk of bowel obstruction. The primary objective of this study was to evaluate whether postoperative pelvic IMRT was associated with less incidence of bowel obstruction, given the improved side effect profile. The secondary objective of this study was to evaluate for other potential risk factors for bowel obstruction in patients with cervical or endometrial carcinoma who receive post-operative pelvic radiation therapy after hysterectomy.

\section{Methods}

After institutional Review Board (IRB) approval was obtained, an institutional database was queried to identify all patients with cervical or uterine cancer who received adjuvant pelvic radiation therapy (RT) after hysterectomy from 2000-2012 at Memorial Sloan Kettering Cancer Center. Only patients who received post-operative pelvic radiation were included; 
patients who received definitive or palliative RT were excluded. Patients with stage IV disease were also excluded.

Medical records including radiation, operative, pathology, chemotherapy, and radiology records were reviewed and clinical data were extracted. Specific clinical information was extracted, such as type of cancer (cervical vs endometrial), type of RT (conventional 3-D RT vs. IMRT), field of RT (pelvic vs. pelvic and para-aortic), body mass index (BMI), adjuvant chemotherapy, abdominal surgeries prior to hysterectomy, type of hysterectomy (simple vs. radical), approach of surgery (minimally-invasive vs. open), and extent of surgical staging (number of pelvic lymph nodes removed). Obesity was defined as a BMI $\geq 30 \mathrm{~kg} / \mathrm{m}^{2}$. The diagnosis of bowel obstruction was made radiographically. Patients who developed bowel obstructions in the setting of recurrent malignancy were excluded.

The technique of IMRT has been previously described. [6,7] In brief, contouring is performed in a manner similar to the Radiation Therapy Oncology Group (RTOG); for lymphatics, obturator, the internal, external, and common iliac vessels and presacral lymph nodes are contoured and two consecutive $7 \mathrm{~mm}$ expansions are applied to create a "pelvic lymph node planning target volume (PTV)" and "presacral PTV." The superior extent of the nodal PTV is the L4-L5 interspace; for a paraaortic field, the superior extent of the nodal PTV is the T12-L1 interspace. The inferior extent of the nodal PTV includes presacral lymph nodes to the level of S3 posteriorly and includes the external iliac nodes to the inguinal ligament. In terms of the "vaginal clinical target volume (CTV)," unlike the RTOG, where motion is accounted for by simulating with both full and empty bladder and incorporating elements of both scans to create ITV, our institution accounts for motion by using generous margins. The vaginal cuff contour (utilizing intravaginal contrast) is expanded initially by $2 \mathrm{~cm}$ to generate the "vaginal cuff CTV," and then an additional $1 \mathrm{~cm}$ axial expansion is applied to create the "vaginal cuff PTV." Weekly imaging was performed.

Clinical follow up consisted of vaginal exams every 3 months and Pap smear and CT chest/ abdomen/pelvis every 6 months for the first 2 years. During years 3-4, patients received exams twice annually and Pap smear/imaging annually. Afterwards, exams and Pap smear were performed annually. All patient follow-up was performed at the primary institution. Grading of toxicity was based on the Common Terminology Criteria for Adverse Events (CTCAE) version 3.0, with the highest grade of any observed toxicity reported for each patient. Follow up and time to event was measured from the date of surgery to the last known visit or death. Standard two-sided statistical tests were used to evaluate for associated risk factors. Kaplan Meier method was used to calculate actuarial rates, with Log rank for comparison between variables. Cox proportional hazards regression analysis test was performed for multivariate analysis.

\section{Results}

A total of 224 patients were identified; all had received post-operative pelvic RT after hysterectomy for cervical or endometrial cancer. Clinical characteristics of the cohort are detailed in table 1 . The median age was 59 years (range, 23-85 years) and the median follow-up time was 67 months. The majority of patients had endometrial cancer $(n=152$, 
$68 \%$ ). The median body mass index (BMI) was $28 \mathrm{~kg} / \mathrm{m}^{2}$ (range, $18-58 \mathrm{~kg} / \mathrm{m}^{2}$ ). Ninety-five (42\%) patients had undergone abdominal surgeries prior to hysterectomy. One hundred and twenty patients (54\%) were treated with intensity modulated radiation treatment (IMRT) whereas 104 patients (46\%) received conventional 3-D RT. The extent of the RT field was as follows; limited to pelvis in 185 patients (83\%), extended to para-aortic region in 39 (17\%). The median RT dose was $50.4 \mathrm{~Gy}$. Adjuvant chemotherapy was given to 163 (73\%) patients. For the 63 patients with cervical cancer it was given as concurrent cisplatin. For the 100 endometrial cancer patients, it was given concurrently in 78 patients and sequentially in 22 .

Table 2 outlines the comparison between these two groups of patients. The IMRT and 3-D RT groups were comparable in terms of age, type of cancer, BMI, prior abdominal surgeries, and number of pelvic lymph nodes removed. The use of adjuvant chemotherapy was more prevalent in the IMRT group than 3-D RT (77\% vs $67 \%, \mathrm{p}=0.09)$. Conversely, more patients underwent an open hysterectomy in the 3-D RT group than in the IMRT group (81\% vs $47 \%,<0.0001)$. The median follow-up was 51 months in the IMRT group and 68 in the 3D RT group. The median dose of radiation was $50.4 \mathrm{~Gy}$ in both groups.

In our cohort, 10 patients (4.5\%) developed a bowel obstruction (BO) following postoperative pelvic RT. The median time to BO was 24 months (range: 7- 65 months). BO was grade 1 (asymptomatic) in 2/224 (0.9\%), grade 2 (conservative management) in $4(1.8 \%)$, and grade $3 \geq \operatorname{in} 4(1.8 \%)$. Overall, the 5-year actuarial rate of $\mathrm{BO}$ was $4.8 \%$ (95\% CI 1.7-7.7). When considering grade $\geq 2 \mathrm{BO}$, the difference was still significant; $6.7 \%$ in the 3 D RT group vs. $0.8 \%$ in the IMRT group, $\mathrm{p}=0.043$.

The rate of BO in the IMRT group was $0.9 \%$ (95\% CI: 0-2.7), compared to 9.3\% (95\% CI: 2.9-15.7) in the 3-D RT group, $\mathrm{p}=0.006$ as shown in figure 1 . The other significant predictor was BMI, for patients with BMI $\geq 30$ the rate was $2.6 \%$ (95\% CI: 0-7.6) compared to $8.3 \%$ (95\% CI: 9-13.7) for BMI <30, p = 0.034. Patient's age, type of cancer, prior abdominal surgeries, whether hysterectomy was open vs minimally invasive, number of pelvic nodes removed, extent of RT field, and the use of chemotherapy didn't significantly impact the rate of bowel obstriction as shown in table 3. On multivariate analysis, only IMRT retained its significance as an independent predictor of less bowel obstruction ( $\mathrm{p}=$ $0.022)$. BMI $\geq 30$ was marginally significant $(p=0.066)$ and having open hysterectomy was not significant $(\mathrm{p}=0.32)$. In the 141 subset of patients who underwent open hysterectomy, 9/84 developed bowel obstruction in those treated with 3-D RT as opposed to 0/57 treated with IMRT ( $\mathrm{p}=0.024)$. In the 83 subset of patients who underwent minimally invasive surgery, there was 1 bowel obstruction in the 63 patients treated with IMRT as opposed to none in the 20 patients treated with 3-D RT $(\mathrm{p}=0.5)$.

\section{Discussion}

In this study, the use of postoperative pelvic IMRT, was associated with significantly less risk of bowel obstruction than 3-D RT ( $0.9 \%$ vs. 9.3\%, p = 0.006). This difference maintained its statistical significance on multivariate analysis $(\mathrm{p}=0.022)$. A rate of $9.3 \%$ for bowel obstruction with 3-D RT, as demonstrated in the current study, is in the range of what has reported in the literature. In GOG 99 randomized trial for intermediate risk endometrial 
cancer, the rate of bowel obstruction was $11.5 \%$ in the postoperative RT arm. [13] In the Landoni randomized trial for early stage cervical cancer, the rate was $5 \%$ for patients treated with postoperative pelvic RT. [14] When limiting the analysis to grade $\geq 2 \mathrm{BO}$, the rate was still significantly less in IMRT group than 3-D RT ( $0.8 \%$ vs. $6.7 \%, \mathrm{p}=0.043)$. Furthermore, none of the patient in the IMRT group developed BO requiring surgical intervention as opposed to 4 in the conventional 3-D RT group.

The IMRT and conventional 3-D RT groups were comparable in terms of age ( $<$ vs. $\geq 60 ; p$ $=0.4$ ), BMI ( $<$ vs. $\geq 30 ; p=0.8$ ), diagnosis (endometrial vs. cervical; $p=0.6$ ), number of pelvic nodes removed ( $<$ vs. $\geq 10 ; p=0.8$ ), and extent of RT field (pelvic vs. extended; $p=$ 0.5 ). More patients in the IMRT group had prior abdominal surgeries ( $47 \%$ vs. $37 ; p=0.16$ ) and more patients received adjuvant chemotherapy ( $77 \%$ vs. $67 \%)$. These differences were not statistically significant and if anything favored the conventional 3-D RT group rather than the IMRT group. Where there was a significant difference related to the type of hysterectomy; significantly more patients in the 3-D RT group had open hysterectomy ( $81 \%$ vs. $47 \%$; $p$ 0.0001). Having an open hysterectomy, however, didn't significantly impact the rate of bowel obstruction on univariate $(p=0.09)$ nor multivariate analysis $(p=0.23)$. This apparent lack of the impact of the route of hysterectomy (open vs minimally invasive) on the rate of bowel obstruction has been demonstrated by others. [15] But perhaps, the strongest evidence comes from the LAP2 randomized trial $(n=2,616)$, where the rate of bowel obstruction was identical at $1 \%$ for both the laparotomy as well as laparoscopy arms. [16]

The potential influence of other treatment-related factors as well as patient's characteristics, on the rate of bowel obstruction, was assessed. As mentioned above, having an open hysterectomy didn't significantly impact the rate of BO. The rate of bowel obstruction was $4 \%$ for patient who had $<10$ pelvic nodes removed compared to $4.9 \%$ for those who had $\geq$ 10 nodes $(\mathrm{p}=0.8)$. In a randomized trial assessing the role of pelvic lymphadenectomy in early stage endometrial cancer $(n=514)$, the rate of bowel obstruction was $1.5 \%$ for patients who underwent lymphadenectomy (median number of pelvic node removed $=26$ ) vs. $1.6 \%$ for those who didn't [16]. This suggests that type and extent of surgery per se, doesn't significantly impact the rate of bowel obstruction. The extent of the radiation field and the use of chemotherapy didn't influence the rate either ( $p=0.19,0.8$, respectively). Regarding patient's characteristics, age, prior abdominal surgeries, and type of cancer (endometrial vs. cervical) didn't impact bowel obstruction rate. For patients with BMI $<30$, the rate was $8.3 \%$ as opposed to $2.6 \%$ for those with $\mathrm{BMI} \geq 30, \mathrm{p}=0.03$ ). This difference was only marginally significant on multivariate analysis, $\mathrm{p}=0.06$. In an analysis of GOG 99 patients who received postoperative pelvic RT, von Gruenigen et al [18] found that patients with BMI <30 were significantly $(\mathrm{p}=0.004)$ more likely to have GI toxicity than those with BMI $\geq 30$. Kizer et al reported on the effect of BMI on complications in patients with cervical cancer who receive definitive chemoradiation therapy.[11] In this study, underweight patients had significantly higher incidence of bowel obstruction $(\mathrm{p}<0.001)$ as well as other GI toxicity such as radiation enteritis and, fistula. [19]

This study is not without its limitations, specifically its retrospective nature with its inherent biases. Thus, one can only state that the use of IMRT was associated with reduction in the rate of bowel obstruction after postoperative pelvic RT in endometrial and cervical cancer 
patients. The debate regarding the advantages and disadvantages of IMRT in terms of cost and need for precise targets delineation will likely continue; [20] this study sought to determine the impact of IMRT on bowel obstruction. To decrease the potential for inherent bias, only patients who were treated since year 2000 were included. In addition, only patients who had their surgery and postoperative RT at our institution were included. The number of events was small, yet they were predominantly in the 3-D RT group. Since the impact of IMRT was also predominantly seen in patients who underwent open hysterectomy, it remains to be determined whether the same magnitude of benefit could be shown in patients undergoing minimally invasive surgery.

In conclusion, the use of postoperative IMRT for cervical and endometrial cancer was associated with significant reduction in the rate of bowel obstruction. This difference maintained its statistical significance on multivariate analysis. Such finding if confirmed by others will help further solidify the benefit of postoperative pelvic IMRT in gynecologic cancers.

\section{Acknowledgments}

Funding: This research was funded in part through the NIH/NCI Cancer Center Support Grant P30 CA008748.

\section{References}

1. Bismar MM, Sinicrope FA. Radiation enteritis. Curr Gastroenterol Rep. 2002; 4(5):361-5. [PubMed: 12228037]

2. Kasibhatla M, et al. Predictors of severe gastrointestinal toxicity after external beam radiotherapy and interstitial brachytherapy for advanced or recurrent gynecologic malignancies. Int $\mathrm{J}$ Radiat Oncol Biol Phys. 2006; 65(2):398-403. [PubMed: 16542793]

3. Birgisson $\mathrm{H}$, et al. Adverse effects of preoperative radiation therapy for rectal cancer: long-term follow-up of the Swedish Rectal Cancer Trial. J Clin Oncol. 2005; 23(34):8697-705. [PubMed: 16314629]

4. Baxter NN, et al. Postoperative irradiation for rectal cancer increases the risk of small bowel obstruction after surgery. Ann Surg. 2007; 245(4):553-9. [PubMed: 17414603]

5. Portelance L, et al. Intensity-modulated radiation therapy (IMRT) reduces small bowel, rectum, and bladder doses in patients with cervical cancer receiving pelvic and paraaortic irradiation. Int $\mathbf{J}$ Radiat Oncol Biol Phys. 2001; 51(1):261-6. [PubMed: 11516876]

6. Shih KK, Milgrom SA, Abu-Rustum NR, Kollmeier MA, Gardner GJ, Tew WP, Barakat RR, Alektiar KM. Postoperative pelvic intensity-modulated radiotherapy in high risk endometrial cancer. Gynecol Oncol. Mar; 2013 128(3):535-9. [PubMed: 23174538]

7. Folkert MR, Shih KK, Abu-Rustum NR, Jewell E, Kollmeier MA, Makker V, Barakat RR, Alektiar KM. Postoperative pelvic intensity-modulated radiotherapy and concurrent chemotherapy in intermediate- and high-risk cervical cancer. Gynecol Oncol. Feb; 2013 128(2):288-93. [PubMed: 23159818]

8. Klopp AH, et al. Hematologic toxicity in RTOG 0418: a phase 2 study of postoperative IMRT for gynecologic cancer. Int J Radiat Oncol Biol Phys. 86(1):83-90. [PubMed: 23582248]

9. Liang Y, Bydder M, Yashar CM, Rose BS, Cornell M, Hoh CK, Lawson JD, Einck J, Saenz C, Fanta P, Mundt AJ, Bydder GM, Mell LK. Prospective study of functional bone marrow-sparing intensity modulated radiation therapy with concurrent chemotherapy for pelvic malignancies. Int J Radiat Oncol Biol Phys. Feb 1; 2013 85(2):406-14. [PubMed: 22687195]

10. Georg P, Georg D, Hillbrand M, Kirisits C, Pötter R. Factors influencing bowel sparing in intensity modulated whole pelvic radiotherapy for gynaecological malignancies. Radiother Oncol. Jul; 2006 80(1):19-26. [PubMed: 16766068] 
11. Chen LA, Kim J, Boucher K, Terakedis B, Williams B, Nickman NA, Gaffney DK. Toxicity and cost-effectiveness analysis of intensity modulated radiation therapy versus 3-dimensional conformal radiation therapy for postoperative treatment of gynecologic cancers. Gynecol Oncol. Mar; 2015 136(3):521-8. [PubMed: 25562668]

12. He S, Gill BS, Heron DE, Kelley JL, Sukumvanich P, Olawaiye AB, Robert P, Edwards RP, Comerci J, Beriwal S. Long-term outcomes using adjuvant pelvic intensity-modulated radiotherapy (IMRT) for endometrial carcinoma. Practical Radiation Oncology. 2016 in press.

13. Keys HM, Roberts JA, Brunetto VL, Zaino RJ, Spirtos NM, Bloss JD, Pearlman A, Maiman MA, Bell JG. Gynecologic Oncology Group. A phase III trial of surgery with or without adjunctive external pelvic radiation therapy in intermediate risk endometrial adenocarcinoma: a Gynecologic Oncology Group study. Gynecol Oncol. Mar; 2004 92(3):744-51. [PubMed: 14984936]

14. Landoni F, Maneo A, Colombo A, Placa F, Milani R, Perego P, Favini G, Ferri L, Mangioni C. Randomised study of radical surgery versus radiotherapy for stage Ib-IIa cervical cancer. Lancet. Aug 23; 1997 350(9077):535-40. [PubMed: 9284774]

15. Mourits MJ, Bijen CB, Arts HJ, et al. Safety of laparoscopy versus laparotomy in early-stage endometrial cancer: a randomised trial. Lancet Oncol. Aug; 2010 11(8):763-71. doi: 10.1016/ S1470-2045(10)70143-1. [PubMed: 20638901]

16. Walker JL, Piedmonte MR, Spirtos NM, Eisenkop SM, Schlaerth JB, Mannel RS, Spiegel G, Barakat R, Pearl ML, Sharma SK. Laparoscopy compared with laparotomy for comprehensive surgical staging of uterine cancer: Gynecologic Oncology Group Study LAP2. J Clin Oncol. Nov 10; 2009 27(32):5331-6. [PubMed: 19805679]

17. Benedetti Panici P, Basile S, Maneschi F, Alberto Lissoni A, Signorelli M, Scambia G, Angioli R, Tateo S, Mangili G, Katsaros D, Garozzo G, Campagnutta E, Donadello N, Greggi S, Melpignano M, Raspagliesi F, Ragni N, Cormio G, Grassi R, Franchi M, Giannarelli D, Fossati R, Torri V, Amoroso M, Crocè C, Mangioni C. Systematic pelvic lymphadenectomy vs. no lymphadenectomy in early-stage endometrial carcinoma: randomized clinical trial. J Natl Cancer Inst. Dec 3; 2008 100(23):1707-16. [PubMed: 19033573]

18. von Gruenigen VE, Tian C, Frasure H, Waggoner S, Keys H, Barakat RR. Treatment effects, disease recurrence, and survival in obese women with early endometrial carcinoma : a Gynecologic Oncology Group study. Cancer. Dec 15; 2006 107(12):2786-91. [PubMed: 17096437]

19. Kizer NT, et al. The effects of body mass index on complications and survival outcomes in patients with cervical carcinoma undergoing curative chemoradiation therapy. Cancer. 117(5):948-56. [PubMed: 20945318]

20. Wagner A, Jhingran A, Gaffney D. Intensity modulated radiotherapy in gynecologic cancers: hope, hype or hyperbole? Gynecol Oncol. Jul; 2013 130(1):229-36. [PubMed: 23632209]

Gynecol Oncol. Author manuscript; available in PMC 2017 October 01. 


\section{Research Highlights}

- $\quad$ IMRT is associated with lower rate of bowel obstruction

- Lower bowel obstruction rate was independent of other prognostic factors

- $\quad$ Results of this study add further credence to the role of IMRT in gynecologic cancers 


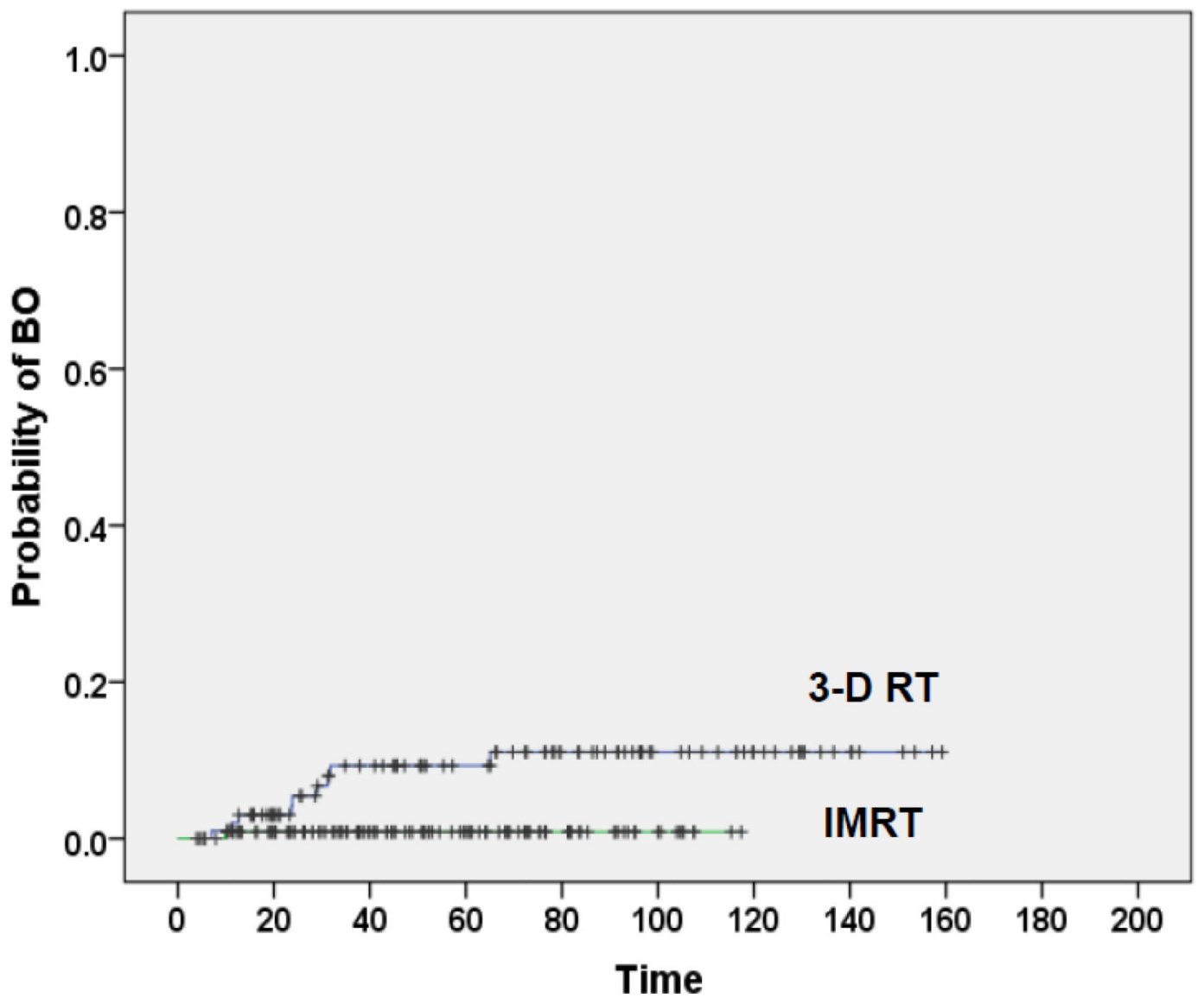

Figure 1.

Probability of bowel obstruction according to radiation type. 3-D RT: 3 dimensional radiation therapy IMRT: Intensity Modulated Radiation Therapy 


\section{Table 1}

Patients and treatment characteristics $(n=224$ patients $)$

\begin{tabular}{|llcc|}
\hline & & n & $\%$ \\
\hline Age at diagnosis (years) & $<60$ & 123 & $55 \%$ \\
& $\succ 60$ & 101 & $45 \%$ \\
\hline BMI (kg/m2) & $<30$ & 130 & $58 \%$ \\
& $\geq 30$ & 94 & $42 \%$ \\
\hline Diagnosis & Endometrial & 152 & $68 \%$ \\
\hline Prior abdominal surgeries & Yes & 72 & $32 \%$ \\
\hline Chemotherapy & No & 95 & $42 \%$ \\
& Yes & 129 & $58 \%$ \\
\hline Type of hysterectomy & No & 163 & $73 \%$ \\
\hline Open & 61 & $27 \%$ \\
\hline RT type & Minimally invasive & 83 & $37 \%$ \\
\hline Extent of RT & IMRT & 120 & $54 \%$ \\
& 3 DRT & 104 & $46 \%$ \\
\hline & Pelvic & 185 & $83 \%$ \\
& Extended field & 39 & $17 \%$ \\
\hline & $<10$ & 61 & $27 \%$ \\
& $\geq 10$ & 163 & $73 \%$ \\
\hline
\end{tabular}

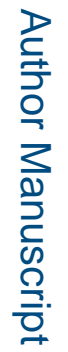

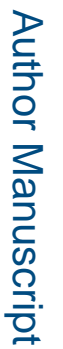

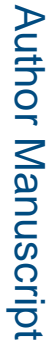


Table 2

Comparison of variables in the IMRT and 3DRT groups

\begin{tabular}{|llccc|}
\hline & & 3DRT (n =104) & IMRT (120) & P value \\
\hline Age (years) & $<60$ & $54(52 \%)$ & $69(57 \%)$ & 0.4 \\
& 260 & $50(48 \%)$ & $51(43 \%)$ & \\
\hline BMI (kg/m2) & $<30$ & $61(59 \%)$ & $69(57 \%)$ & 0.87 \\
& $\geq 30$ & $43(41 \%)$ & $51(43 \%)$ & \\
\hline Type of cancer & Endometrial & $72(69 \%)$ & $80(67 \%)$ & 0.69 \\
& Cervical & $32(31 \%)$ & $40(33 \%)$ & \\
\hline Prior abdominal surgeries & Yes & $39(37 \%)$ & $56(47 \%)$ & 0.16 \\
& No & $65(63 \%)$ & $64(53 \%)$ & \\
\hline Chemotherapy & Yes & $70(67 \%)$ & $93(77 \%)$ & 0.09 \\
& No & $34(33 \%)$ & $27(23 \%)$ & \\
\hline Type of hysterectomy & Open & $84(81 \%)$ & $57(47 \%)$ & $<0.0001$ \\
& Minimally invasive & $20(19 \%)$ & $63(53 \%)$ & \\
\hline Extent of RT & Pelvic & $84(81 \%)$ & $101(84 \%)$ & 0.5 \\
& Extended field & $20(19 \%)$ & $19(16 \%)$ & \\
\hline \# of pelvic LN dissected & $<10$ & $29(28 \%)$ & $32(27 \%)$ & 0.84 \\
& $\geq 10$ & $75(72 \%)$ & $88(73 \%)$ & \\
\hline
\end{tabular}

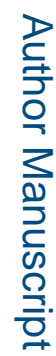

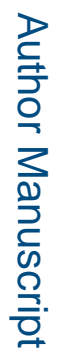

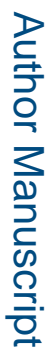


Table 3

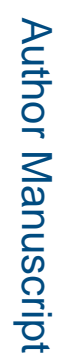

Bowel obstruction according to variables

\begin{tabular}{|c|c|c|c|}
\hline Variables & & $5 \mathrm{yr}$ rates $(95 \% \mathrm{CI})$ & $\mathbf{p}$ \\
\hline \multirow[t]{2}{*}{ Age } & $<60$ & $2.7(0-5.9)$ & 0.26 \\
\hline & 260 & $7.4(1.6-13.2)$ & \\
\hline \multirow[t]{2}{*}{ BMI } & $<30$ & $8.3(2.09-13.7)$ & 0.03 \\
\hline & $\geq 30$ & $2.6(0-7.6)$ & \\
\hline \multirow[t]{2}{*}{ IMRT } & Yes & $0.9(0-2.7)$ & 0.006 \\
\hline & No & $9.3(2.9-15.7)$ & \\
\hline \multirow[t]{2}{*}{ Type of cancer } & Endometrial & $5.5(4.2-9.7)$ & 0.8 \\
\hline & Cervical & $5.9(0-12.7)$ & \\
\hline \multirow[t]{2}{*}{ RT field } & Pelvic & $3.8(0.8-6.8)$ & 0.19 \\
\hline & Extended field & $9.4(0-19.8)$ & \\
\hline \multirow[t]{2}{*}{ Prior abdominal surgeries } & Yes & $4.8(0.2-9.4)$ & 0.85 \\
\hline & No & $4.7(0.5-8.9)$ & \\
\hline \multirow[t]{2}{*}{ \# of pelvic LN dissected } & $<10$ & $4(0-9.6)$ & 0.80 \\
\hline & $\geq 10$ & $4.9(1.3-8.5)$ & \\
\hline \multirow[t]{2}{*}{ Chemotherapy } & Yes & $4.4(0.8-8)$ & 0.81 \\
\hline & No & $5.7(0-12.1)$ & \\
\hline \multirow[t]{2}{*}{ Open hysterectomy } & Yes & $6.6(2-11.2)$ & 0.09 \\
\hline & No & $1.2(0-3.6)$ & \\
\hline
\end{tabular}

